\title{
“BULLYNG Y LOS FACTORES DE RIESGO EN LOS ESTUDIANTES DEL COLEGIO NACIONAL NUESTRA SEÑORA DE LAS MERCEDES DE ICA" - 2013
}

"Bullyng and risk factors in National College Students our Lady of Mercy of Ica" - 2013

\author{
Cristina Uribe Rosas ${ }^{1, a, b}$ Alejandrina Bertha Pebes Mendoza ${ }^{2, a, b}$ \\ ${ }^{1}$ Facultad de Enfermería. Universidad Nacional "San Luis Gonzaga" de Ica, Perú, ${ }^{2}$ Hospital Regional de Ica - Perú \\ ${ }^{a}$ Licenciada en Enfermería ${ }^{b}$ Doctora en Salud Pública.
}

\section{RESUMEN}

Objetivo.- Determinar la influencia de los factores de riesgo (Funcionabilidad familiar) del bullyng en las alumnas del Colegio Nacional "Nuestra Señora de las Mercedes de Ica" - 2013. Material y Métodos.- La investigación asume un diseño no experimental, de tipo descriptivo - Correlacional.

Resultados.- Los instrumentos utilizados fueron el Auto test Cisneros y el Apgar familiar. El nivel de bullying acumulado es de $36,5 \%$. El bullying hallado según género es de $75,2 \%$ mujeres. No me hablan con un $18,75 \%$, un $17,11 \%$ refieren que lo llaman por sobre nombres, por otro lado el $8,55 \%$ manifiestan que se ríen cuando se equivocan, en un 5,92\% optan por la modalidad de, Cambian el significado de todo lo que digo, No me dejan que participe me excluyen 5,59\%, y Me odian si razón 5,26 . Los niveles de funcionalidad familiar evidencian que el $27,6 \%$ presenta una buena función familiar, el 29,7\% una disfunción leve, el 24,3\% una disfunción moderada y el 18,4\% una disfunción severa, es decir las alumnas de la Institución Educativa Nuestra Señora de las Mercedes se encuentra con Disfuncionalidad familiar leve. También se halló que existe una correlación entre acoso acolar y los factores de riesgo (funcionalidad familiar) $(0,031 p<0,05)$. La correlación entre las dos variables reportó un índice de 0,240 p> 0,05, es decir el índice global de acoso no se encuentra relacionado al sexo de la alumna. Conclusiones.- En base a los datos de las investigaciones nacionales, no podemos precisar, si este fenómeno está en aumento o está disminuyendo. Sin embargo los resultados hallados en esta investigación nos permiten afirmar que los porcentajes de niñas victimas del bullying son del $36,5 \%$.

Palabras claves: Escolares, bullying, factores de riesgo (funcionalidad familiar).

\section{SUMMARY}

Objective.- Determine the influence of risk factors (family Functionality ) of bullyng in the students of "Nuestra Señora of the Mercedes of Ica " National College - 2013.

Material and Methods.- Research assumes a non- experimental design, descriptive - correlational. Results.- Instruments used were the Auto test Cisneros and family Apgar. The cumulative level of bullying is $36,5 \%$. The bullying found by gender is $75,2 \%$ female. I do not speak with $18,75 \%$ a $17,11 \%$ reported they call over names , on the other hand, the $8.55 \%$ state that they laugh when they are wrong , a 5,92\% choose the mode, they change the meaning of everything I say, do not let me participate exclude me 5,59\%, and if reason I hate 5,26. Levels of family functioning show that $27,6 \%$ have a good family function, $29,7 \%$ mild dysfunction , $24,3 \%$ moderate dysfunction and $18,4 \%$ severe dysfunction, ie the students of School Our Lady of Mercy is familiar with mild Dysfunctionality. We also found a correlation between acolar harassment and risk factors (family functioning) $(0,031<p 0,05)$. The correlation between the two variables reported a rate of $0,240 p>$ 0,05 , ie the overall rate of harassment is not related to the sex of the student. Conclusions. Based on national research data, we can not specify if this phenomenon is increasing or declining. However, the results found in this study allow us to state that the percentages of girls bullying victims are $36,5 \%$.

Keywords: School, bullying, risk factors (family functioning). 


\section{INTRODUCCIÓN}

El bullying, es una de las formas de violencia que se dan entre los escolares y que por ser un fenómeno socioeducativo, debe ser motivo de preocupación en las autoridades educativas y padres de familia, por las consecuencias negativas que este tipo de conductas ocasionan en los niños que sufren de este tipo de maltratos. Este es el motivo fundamental por el cual se Hizo este tipo de investigaciones, para conocer y dar a conocer la magnitud real de estos hechos y así ir rompiendo el círculo del silencio. Así mismo debemos romper el patrón cultural de concebir como "algo normal" estas situaciones de intimidación, violencia, exclusión, discriminación como propio de los niños $y$ de la cotidianidad de los establecimientos educativos (1).

Desde esta perspectiva el bullying es un problema sistémico, por lo que toda persona o institución que está involucrada en el cuidado y desarrollo de los niños y adolescentes (familia, profesores, auxiliares, directores, orientadores, tutores, psicólogos psiquiatras, psicopedagogos, amigos, víctimas, observadores, profesoras de inicial y gobiernos locales) tienen alguna responsabilidad compartida para frenar este mal que afecta a lo más valioso de nuestros niños: su autoestima, dañando así su presente y su futuro.

Las diversas investigaciones sobre el bullying, señalan como causas de origen de este fenómeno a los factores familiares, personales, sociales, económicos y escolares. Bajo este contexto, en el Perú existe un gran número de familias desestructuradas, pudiendo ser el elemento clave en la génesis de las conductas violentas, por la falta de una adecuada comunicación, reglas de convivencia y la ausencia de los progenitores.

Al respecto, según la Encuesta Nacional de Hogares, realizado por el Instituto Nacional de Estadística e Informática (INEI, 2008) el $76,9 \%$ de hogares es conducido por un varón $y$ el $23,1 \%$ por una mujer. Es oportuno resaltar que este $23,1 \%$ de familias, que son conducidas por una mujer, se deba a razones de divorcio, abandono, viudez y que tienen que asumir la difícil tarea de cuidar y educar a los hijos (2).

Otra probable causa son los índices de violencia familiar. Según datos elaborados por el Movimiento Manuela Ramos (2010), el departamento que registró mayor violencia familiar durante el primer semestre del 2009, es Lima con el 43,08\% (20,940 casos denunciados).

En nuestro país un caso que merece la atención es lo ocurrido en Huánuco el 23 de Abril del 2010, con la muerte de un escolar de 7 años, por sus dos compañeros de 8 y 10 años, quienes le golpearon al momento de la salida del colegio 3 casos como estos, son los que obligan a realizar este tipo de investigaciones, para poder tomar medidas correctivas y facilitar una convivencia de paz.

Después de lo anterior expuesto, esta investigación nace como una necesidad de promocionar la salud mental de las estudiantes de la Institución Educativa "Nuestra Señora de las Mercedes" Ica 2013, ya que la labor del profesional de Enfermería es la atención integral de la persona como un ser Biopsicosocial. Los resultados obtenidos en esta investigación servirán para elaborar estrategias y programas de convivencia escolar, con la finalidad de fomentar la integración y cultivar los valores entre los escolares (3).

Por lo tanto el objetivo de esta investigación fue determinar la influencia de los factores de riesgo (Funcionabilidad familiar) del bullyng en las alumnas del Colegio Nacional "Nuestra Señora de las Mercedes de Ica" - 2013

\section{MATERIALES Y MÉTODOS.}

El tipo de investigación utilizado corresponde al Descriptivo - Correlacional. Descriptivo porque nos permite describir y medir las dos variables del estudio: bullying y Factores de riesgo (funcionalidad familiar). Asimismo es Correlacional porque nos permite conocer las 
relaciones existentes entre el bullying $y$ Factores de riesgo (la funcionalidad familiar). El tipo de diseño que empleamos para alcanzar los objetivos de esta investigación corresponde a la investigación No Experimental. Bajo este enfoque No Experimental, el diseño que consideramos apropiado para esta investigación es la Transversal o Transeccional, ya que los datos son recolectados en un solo momento o tiempo único, buscando describir las variables del estudio y analizar su incidencia e interrelación. Los instrumentos que se han utilizado son el Autotest de Cisneros de Acoso Escolar y el Cuestionario de Apgar Familiar (4).

\section{RESULTADOS}

El nivel de bullying acumulado es de $36,5 \%$. El bullying hallado según género es de $75,2 \%$ mujeres. No me hablan con un $18,75 \%$, un
$17,11 \%$ refieren que lo llaman por sobre nombres, por otro lado el $8,55 \%$ manifiestan que se ríen cuando se equivocan, en un $5,92 \%$ optan por la modalidad de, Cambian el significado de todo lo que digo, No me dejan que participe me excluyen $5,59 \%$, y Me odian si razón 5,26. Los niveles de funcionalidad familiar evidencian que el $27,6 \%$ presenta una buena función familiar, el $29,7 \%$ una disfunción leve, el 24,3\% una disfunción moderada y el 18,4\% una disfunción severa, es decir las alumnas de la Institución Educativa Nuestra Señora de las Mercedes se encuentra con Disfuncionalidad familiar leve. También se halló que existe una correlación entre acoso acolar y los factores de riesgo (funcionalidad familiar) $(0,031$ $p<0,05)$. La correlación entre las dos variables reportó un índice de 0,240 p> 0,05, es decir el índice global de acoso no se encuentra relacionado al sexo de la alumna.

TABLA 1. Grado de estudios de las alumnas del nivel secundario del Colegio Nacional "Nuestra Señora de las Mercedes de Ica

\begin{tabular}{llcc}
\hline \multicolumn{1}{l}{ Edad de las } & \multicolumn{2}{c}{ Frecuencias } \\
alumnas & $\mathrm{f}_{\mathrm{i}}$ & $\mathrm{p}_{\mathrm{i}} \%$ \\
\hline 12 años & 35 & 11,5 \\
13 años & 65 & 21,4 \\
14 años & 63 & 20,8 \\
15 años & 46 & 15,1 \\
16 años & 59 & 19,4 \\
17 años & 31 & 10,2 \\
$\overline{\boldsymbol{X}}=14.5 ;$ & 05 & 01,6 \\
\cline { 2 - 4 } 18 años & TOTAL & 304 & 100,0 \\
\hline
\end{tabular}

TABLA 2. Grado de instrucción de las alumnas del nivel secundario del Colegio Nacional "Nuestra Señora de las Mercedes de Ica

\begin{tabular}{|c|c|c|c|}
\hline $\begin{array}{l}\text { GRADO DE } \\
\text { INSTRUCCIÓN }\end{array}$ & POBLACIÓN & MUESTRA & $\%$ \\
\hline Primer Año & 98 & 57 & $18,8 \%$ \\
\hline Segundo Año & 146 & 75 & $24,7 \%$ \\
\hline Tercer Año & 148 & 69 & $22,6 \%$ \\
\hline Cuarto Año & 143 & 47 & $15,5 \%$ \\
\hline Quinto Año & 175 & 56 & $18,4 \%$ \\
\hline TOTAL & 710 & 304 & $100 \%$ \\
\hline
\end{tabular}


TABLA 3. Modalidades más Frecuentes de Bullying en las alumnas del nivel secundario del Colegio Nacional "Nuestra Señora de las Mercedes de Ica

\begin{tabular}{|c|c|c|}
\hline \multirow{2}{*}{ ITEM } & \multicolumn{2}{|c|}{ Frecuencia } \\
\hline & $f_{i}$ & $\mathrm{p}_{\mathrm{i}} \%$ \\
\hline No me hablan & 57 & 18,75 \\
\hline No me dejan hablar & 24 & 7,89 \\
\hline No me dejan jugar con ellos & 29 & 9,54 \\
\hline Me llaman por sobrenombres & 52 & 17,11 \\
\hline Me amenazan para que haga cosas que no quiero & 10 & 3,29 \\
\hline Me obligan a hacer cosas que están mal & 6 & 1,97 \\
\hline No me dejan que participe, me excluyen & 17 & 5,59 \\
\hline Me obligan a hacer cosas que me hacen sentir mal & 10 & 3,29 \\
\hline Rompen mis cosas a propósito & 4 & 1,32 \\
\hline Roban mis cosas & 5 & 1,64 \\
\hline Les dicen a los demás que no hablen conmigo & 4 & 1,32 \\
\hline Hacen gestos de burla o desprecio hacia mi & 12 & 3,95 \\
\hline Me pegan: puñetazos, patadas, etc. & 5 & 1,64 \\
\hline Se ríen de mi cuando me equivoco & 26 & 8,55 \\
\hline Me pegan con objetos & 8 & 2,63 \\
\hline Cambian el significado de lo que digo & 18 & 5,92 \\
\hline Se meten conmigo por mi forma de ser & 14 & 4,61 \\
\hline Se meten conmigo por ser diferente (raza, condición socio económica, género, etc.) & 9 & 2,96 \\
\hline Van contando por ahí mentiras acerca de mi & 13 & 4,28 \\
\hline Me zarandean o empujan para intimidarme & 4 & 1,32 \\
\hline Intentan que me castiguen & 11 & 3,62 \\
\hline Me desprecian & 5 & 1,64 \\
\hline Me amenazan con armas & 3 & 0,99 \\
\hline Amenazan con dañar a mi familia & 2 & 0,66 \\
\hline Intentan perjudicarme en todo & 6 & 1,97 \\
\hline Me odian sin razón & 16 & 5.26 \\
\hline TOTAL DE ALUMNAS ENCUESTADAS & $\begin{array}{l}30 \\
4\end{array}$ & 100,0 \\
\hline
\end{tabular}

TABLA 4

Factores de riesgo (Apgar familiar) en las alumnas del nivel secundario de la Institución Educativa "Nuestra Señora de las Mercedes"

\begin{tabular}{lll}
\hline \multirow{2}{*}{ APGAR FAMILIAR } & \multicolumn{2}{l}{ Frecuencias } \\
\cline { 2 - 3 } & $\mathrm{fi}$ & $\mathrm{pi} \%$ \\
\hline Buena función familiar & 84 & 27,6 \\
Disfunción familiar leve & 90 & 29,7 \\
$\begin{array}{l}\text { Disfunción familiar } \\
\text { moderada }\end{array}$ & 74 & 24,3 \\
Disfunción familiar severa & 56 & 18,4 \\
\hline TOTAL & 304 & 100.0 \\
\hline
\end{tabular}

\section{DISCUSIÓN.}

El presente estudio se realizó tomando en cuenta el índice de acoso escolar medida a través del auto test de Cisneros, en el cual se observa el cuadro $\mathrm{N}^{\circ} 1$ que el grupo de edad más frecuente de las alumnas encuestadas corresponden a los que tienen 13 años de edad (5).
El maltrato que se da entre escolares, dentro del contexto educativo, es internacionalmente conocido como bullying y es una modalidad de violencia escolar que está presente en las escuelas de todos los niveles socioeconómicos; bajo distintas modalidades. Es una realidad multiforme, silenciosa, cambiante y que afecta a todos los escolares. 
Las diversas investigaciones indican que el bullying es un fenómeno complejo de naturaleza psicosocial que constituye un problema de salud pública. Los resultados hallados en esta investigación evidencian la existencia de un $36.5 \%$ (porcentaje acumulado medio, casi alto, alto, muy alto) de bullying en la muestra estudiada y son consistentes con la literatura de donde surgen diferentes formas de bullying, $y$ las investigaciones hechas en nuestro país y el extranjero coinciden con los datos obtenidos en este estudio. Así, Oliveros y Barrientos (2007) reportaron un índice de 54,7\% de acoso escolar en un colegio de Lima. Oliveros y Figueroa (2009) hallaron la existencia de $47 \%$ de acoso escolar en los colegios de Lima Metropolitana y algunas provincias.

En lo referente a las modalidades más frecuentes de bullying que se hallaron en esta investigación, podemos afirmar que los modos más predominantes son: No me hablan con un $18,75 \%$, un $17,11 \%$ refieren que lo llaman por sobrenombres, por otro lado el $8,55 \%$ manifiestan que se ríen cuando se equivocan, en un 5,92\% optan por la modalidad de, Cambian el significado de todo lo que digo, No me dejan que participe me excluyen $5,59 \%$, y Me odian si razón 5,26\%. Estos datos coinciden con lo hallado por otros investigadores. Así, Oliveros y Barrientos (2007) hallaron que la modalidad más frecuente de acoso escolar en las estudiantes de Lima Metropolitana era el Hostigamiento verbal (poner sobrenombres o apodos, etc.) predominado con $38,7 \%$ (6).

Así, en la actualidad el poner sobrenombres es algo muy común en todos los niveles de la sociedad. Pero lo que pasa desapercibido es el efecto negativo que este tipo de maltratos puede ocasionar en las víctimas que son objeto de este tipo de agresiones y peor aún en los contextos escolares. La adolescente acosadora con alguna frecuencia replica la conducta que se desarrolla en su hogar. La acosadora según las diversas investigaciones lidera un grupo, se presenta como el más fuerte y establece el sistema de valores de quienes lo admiran y festejan lo que hace. Frente a estos hechos muchos profesores y padres de familia no hacen nada al respecto y por tanto la acosadora asume un estilo de vida y una forma de dominar a los demás que, de llegar a ser exitosos para él, lo convertirán en un acosador adulto.

En lo referente a la intensidad del bullying, en esta investigación se determinó que el 51.3\% de la muestra estudiada es víctima frecuente de este tipo agresiones y maltratos.

En cuanto a los resultados obtenidos por los componentes del Auto-test Cisneros, los resultados evidencian que el $51 \%$ de la muestra de estudio es víctima de desprecioridiculización, de coacciones 23,7\%, de restricción-comunicación $50 \%$, de agresiones $32,5 \%$, de intimidación-amenazas $50 \%$, de exclusión-bloqueo social 63,2\%, de hostigamiento verbal $52,9 \%$ y de robos $25,4 \%$, frente a sus compañeros de estudio. Estos datos guardan relación con las investigaciones de Piñuel y Oñate (2007) (7).

Los resultados indican un alto contenido de violencia y agresiones entre compañeras de escuela. Este tipo de conductas son perjudiciales para la salud emocional de los escolares porque dañan su autoestima y afectan su rendimiento académico. Otro aspecto que llama la atención, son los resultados del componente de robos, el $25,4 \%$ de la muestra de estudio manifiesta que es víctima de robos dentro de las aulas de clase y esto pone en evidencia que el sistema de valores dentro de las familias de los escolares de la institución educativa no está bien.

En los resultados de Arellano N. Chirinos Y. López y Sánchez. (Octubre 2007) se rescata que las manifestaciones relacionadas con violencia y maltrato físico y psicológico se presentan con relativa frecuencia, vistas separadamente podría interpretarse como una situación general poco problemática. No obstante, si se consideran en conjunto (algunas veces - casi siempre) prácticamente 
todas están por encima del 50\%, lo cual debería ser una señal de alerta para el colectivo profesoral. Se observa que la violencia o maltrato verbal (insultar, hablar mal, poner apodos o sobrenombres) presenta altos porcentajes por encima de la agresión física (golpear) lo cual supone una revisión a los procesos de comunicación en la institución (8).

Los resultados obtenidos sobre los factores de riesgo (funcionalidad familiar) nos permiten determinar que el $27,6 \%$ de las niñas provienen de familias cuya funcionalidad es muy buena y el $29,7 \%$ de familias con una disfuncionalidad leve y un $24,3 \%$ presenta una disfuncionalidad moderada en tanto que el $18,4 \%$ representa una disfunción familiar severa. Estos datos pueden justificarse ya que los niños que asisten al colegio donde se llevó la investigación provienen de diversos sectores de la Ciudad de Ica (por ser un colegio nacional acoge a niños de familias en situaciones de pobreza y con conflictos familiares que viven en las zonas populosas de Ica, La Palma, Acomayo, Calle Tumbes, Santiago etc.). $Y$ bajo esta realidad es muy usual que en los hogares de estas niñas existan dificultades de orden económico, afectivo y familiares.

Los análisis por ítems, sobre la funcionalidad familiar nos permiten precisar que el $32,9 \%$ de la muestra de estudio, presenta dificultades en el área de apoyo entre sus miembros, el $45,1 \%$ presenta dificultades en el área de participación, el 31.6\% manifiesta tener familias muy rígidas y autoritarias, el $44,4 \%$ no se siente satisfecho con la ayuda que recibe de su familia en el área de los afectos y emociones, el $38,4 \%$ no está satisfecho en el área recreacional e integración familiar (9).

Estos resultados evidencian que las familias de la institución educativa "Nuestra Señora de las Mercedes" de Ica no satisfacen de manera adecuada las demandas y necesidades de sus hijos en las diferentes áreas de la dinámica familiar. Resulta oportuno también señalar que la insatisfacción familiar que sienten los escolares con respecto a su familia, pueda ser un causal para generar situaciones de bullying escolar (10).

Según Toledo Jofre M. quien estudio la relación entre intimidación, clima escolar y rendimiento (Santiago de Chile 2009) se comporta de forma diferentes entre las

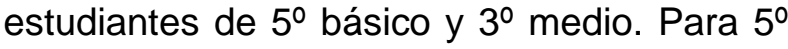
básico, la intimidación influye la variabilidad del clima escolar y del rendimiento en forma directa y para $3^{\circ}$, la intimidación afecta la variabilidad del rendimiento sólo por medio del clima escolar, es decir, de manera indirecta. Sin embardo en nuestra investigación el cual el bullying la relacionamos con los factores de riesgo (funcionabilidad familiar) se encuentra una relación significativa entre ambas variables.

Los resultados obtenidos sobre la correlación entre bullying y los factores de riesgo (funcionalidad familiar), demuestran que existe una correlación entre ambas variables. Sin embargo esto significa que las variables estudiadas no necesariamente guardan una relación directa, es decir que los niños que provienen de familias estables o funcionales estarán más preparados para enfrentar situaciones de bullying y maltrato entre sus compañeros. En consecuencia se espera que los niveles de bullying sean menores.

Para resolver el problema fundamental, sus objetivos y las hipótesis, se han utilizado para la contrastación de esta última, la aplicación de los instrumentos, y refrendados a través del uso del chi cuadrado, llegando a las siguientes

\section{CONCLUSIONES:}

1. Los resultados de esta investigación evidencian que existe una relación entre bullying y los factores de riesgo (funcionalidad familiar) en las estudiantes de la muestra, evidenciando que los niveles altos de bullying, se deben a niveles bajos de funcionalidad familiar. 
2. En base a los datos de las investigaciones nacionales, no podemos precisar, si este fenómeno está en aumento o está disminuyendo. Sin embargo los resultados hallados en esta investigación nos permiten afirmar que los porcentajes de niñas victimas del bullying son del $36,5 \%$.

3. Los niveles del bullying en las estudiantes de la Institución Educativa "Nuestra Señora de las Mercedes" Ica 2013, son muy bajas.

4. Las modalidades más frecuentes de bullying, en las estudiantes de la Institución Educativa "Nuestra Señora de las Mercedes" Ica 2013, corresponden La modalidad más frecuente es exclusión bloqueo social, Hostigamiento verbal, Desprecio - Ridiculización, Restricción comunicación y del mismo porcentaje el componente Intimidación - Amenazas.

5. Las investigaciones revisadas, señalan que los modelos de crianza y sistemas familiares son factores de riesgo para la generación de conductas hostiles por parte de las alumnas. En este sentido, los datos hallados en esta investigación, evidencian que el $18,4 \%$ de la muestra de estudio proviene de familias con disfuncionalidad severa.

6. También es pertinente considerar la influencia del factor familiar en lo relativo a falta o ausencia de comunicación, ya que los datos obtenidos en esta investigación indican porcentajes no alarmantes; pero sí, significativos de las carencias en las áreas de adaptación, participación, ganancia, afecto y recursos.

\section{CORRESPONDENCIA:}

Dra. Cristina Uribe Rosas

Correo electrónico: Esther_uribe_27@hotmail.com

\section{REFERENCIAS BIBLIOGRÁFICAS.}

1) Aproximación al fenómeno bullying [base de datos en [Internet]. España: Acosomoral.org; 2006. [fecha de acceso 5 de junio de 2013]. URL disponible en: http://www.acosomoral.org/pdf/Tresgallo/tr esgallo5.PDF
2) Oliveros M. Incidencia y factores de riesgo de la intimidación (bullying) en un colegio particular de Lima, Perú; 2007 [Internet]. Rv. Peruana de Pediatría; 2007. [fecha de acceso 5 de junio de 2013]. URL disponible en: http://revistas.concytec. gob.pe/pdf/rpp/v60n3/a03v60n3.pdf

3) Landázuri V. Asociación entre el rol de agresor y el rol de víctima de intimidación escolar, con la autoestima y las habilidades sociales de adolescentes de un colegio particular mixto de lima [Internet]. Perú: Artículos originales; 2007. [fecha de acceso 10 de junio de 2013]. URL disponible en: http://www.upch. edu.pe/fapsi/rph/NUMERO/Landazuri.pdf

4) Oliveros M. Intimidación en colegios estatales de secundaria del Perú [Internet]. Perú: Revista Peruana de Pediatría; 2009. [fecha de acceso 15 de junio de 2013]. URL disponible en: http://revistas.concytec.gob.pe/ pdf/rpp/v62n2/a04v62n2.pdf

5) Romaní F. Auto-reporte de victimización escolar y factores asociados en escolares peruanos de educación secundaria, año 2007. [Internet] Perú: Revista peruana de Epidemiologia; 2009. [fecha de acceso 24 de junio de 2013]. URL Disponible en: http://www.sisbib.unmsm.edu.pe/BVRevist as/epidemiologia.

6) Arellano N. Los tipos de maltrato entre iguales [base de datos en ( Internet]. Argentina: Todo sobre mediación; 2013. [fecha de acceso 6 de julio de 2013]. URL disponible en: http://www.todosobre mediacion.com/articulos/colaboraciones/6 0-los-tipos-de-maltratos-entre-iguales

7) Piñuel I. Autotest Cisneros. [Internet]. España: Instituto de Innovación Educativa y Desarrollo Directivo; 2005. [fecha de acceso 6 de julio de 2013]. URL disponible en: http://www.internenes. com/acoso/docs/autotest-1.pdf 
8) Oliveros $M$, Incidencia y factores de riesgo de la intimidación (bullying) en un colegio particular de Lima-Perú, 2007 [en línea]. Perú: Revista Pediátrica; 2007. [fecha de acceso 10 de julio de 2013]. URL disponible en: http://www.observatorio peru.com.

9) Jiménez A. El maltrato entre escolares (Bullying) en el primer ciclo de Educación Secundaria Obligatoria: valoración de una intervención a través de medios audiovisuales [Internet]. España: Universidad de Huelva; 2007. [fecha de acceso 10 de julio de 2013]URL disponible en: http://rabida.uhu.es/ dspace/handle/10272/62
10) Arias L. Apgar Familiar [Internet]. Colombia: Oocities.org; 2008. [fecha de acceso 10 de mayo de 2013]. URL disponible en: http://www.oocities.org/ trabajocomunitario6/index/APGAR.pdf.

Recibido: 20/02/14

Aprobado para Publicación: 25/05/14 